\title{
A critical review of reduced one-dimensional beam models of piezoelectric composite beams
}

\author{
Luca Luschi ${ }^{1}$, Giuseppe lannaccone ${ }^{1}$, Francesco Pieri ${ }^{1}$
}

\begin{abstract}
Simplified one-dimensional models for composite beams with piezoelectric layers, which are intrinsically threedimensional structures, are important for many applications, including piezoelectric energy harvesters. To reduce the dimensionality of the system, assumptions on the stress/strain state in the transverse direction are typically made. The most common are those of null transverse stress (NTS), used for narrow beams, null transverse deformation (NTD), used for wide beams, and continuous interface strain, suited for thin piezoelectric layers (we call this assumption Thin Film Continuous or TFC).

We show that the models based on these assumptions are often used uncritically for beam geometries for which large errors may result. In particular, NTS fails even for narrow beams if the thickness is much smaller than the beam width. We give clear geometric criteria that, for any geometry, allow the selection of the most accurate model among the three. We also develop a single, unified beam equation encompassing the three models and compare the analytical results from this equation with Finite Element simulations over a wide range of beam lengths, widths, and layer thicknesses. The selection criteria and the unified beam equation form a valuable tool for fast and accurate design of composite piezoelectric beams.
\end{abstract}

\section{Keywords}

piezo films, piezoelectric beam, energy harvesting, Micro-Electro-Mechanical Systems, MEMS

\section{Introduction}

Piezoelectric actuation and detection of beams is used in a wide range of applications. Cantilever harvesters have been proposed both as micro-engineered solutions (Erturk and Inman 2009; Karami et al. 2011; Reissman et al. 2016; Rafique and Bonello 2010) and based on Micro-ElectroMechanical Systems (MEMS) technology (Jackson et al. 2017; Olszewski et al. 2017; Jia and Seshia 2016; Lei et al. 2014; Chen et al. 2006; Isarakorn et al. 2011). Resonant cantilevers exploiting the gravimetric principle have been employed as gas sensors (Ivaldi et al. 2011; Karabacak et al. 2010), and biosensors (Shin et al. 2008). Gas sensing can also be performed with photoacoustic detectors where cantilevers are used as microphones (Ledermann et al. 2004; Glauvitz et al. 2012). Other possible applications include atomic force microscopy (Korayem et al. 2017; Quenzer et al. 2011; Shibata et al. 2004), RF switches (Mahameed et al. 2008; Wunnicke 2009), accelerometers (Hewa-Kasakarage et al. 2013; Hindrichsen et al. 2010) and actuators (Dong et al. 2007). A survey of the literature also shows that MEMS technology is used in a growing number of devices, for which the beam geometries (i.e.

Prepared using sagej.cls [Version: 2017/01/17 v1.20] width, thickness, etc.) are scattered over a larger range than for micro-engineered devices (Fig. 2).

A vast literature about the modelling of piezoelectric composite beams exists. However, we observed that for a number of these models the actual limits of accuracy as a function of the beam geometry are not investigated, so that picking the model which gives accurate results for the problem at hand-if any exists-is obvious. The literature is also affected by the use of disparate, and sometimes inconsistent, notations, which make comparison among the different models difficult.

Because of these hurdles, several works choose an inadequate model to predict the behavior of actual devices, making large errors as a result. The present paper addresses these problems through the development of a generalized beam model which includes most cases presented in the literature, and the definition of clear criteria to decide the model which provides the best approximation for each case, while also pointing to the use of incoherent notations which

${ }^{1}$ Dipartimento di Ingegneria dell'Informazione, Università di Pisa, Pisa, Italy

Corresponding author:

Francesco Pieri, Via Caruso 16, 56122 Pisa, Italy

Email: francesco.pieri@unipi.it 
may hinder the understanding of a few existing works.

Although an exact description of piezoelectric composite beams cannot neglect the 3D behavior of the piezoelectric layers, simplified 1D formulations can provide sufficient accuracy in many circumstances, with the advantage of providing practical tools for design and optimization purposes. 1D models are obtained by choosing simplifying assumptions on the cross-sectional deformations of the composite structure, and have the further advantage of being embeddable in the classic Euler-Bernoulli (EB) or Timoshenko beam (TB) theories (Hagedorn and DasGupta 2007).

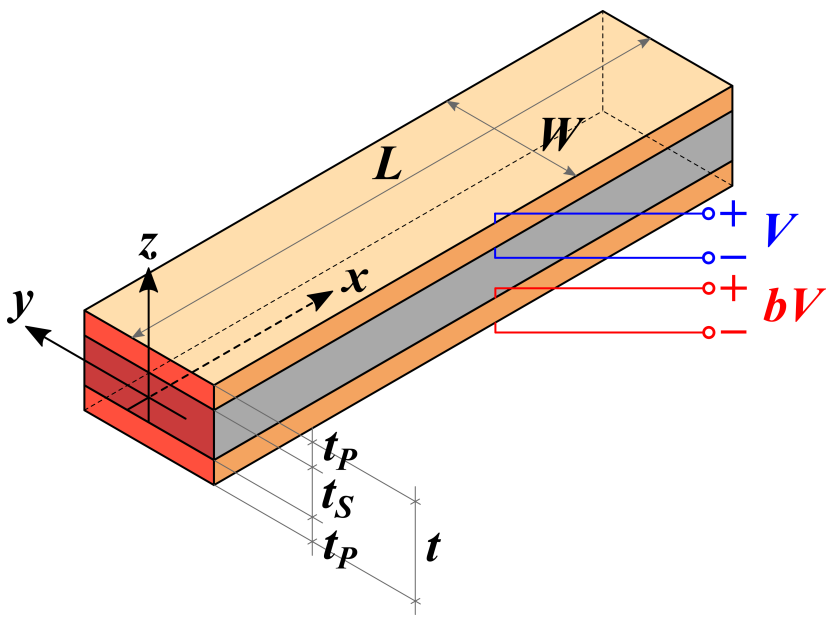

Figure 1. piezoelectric triple layer cantilever bimorph. The piezoelectric material is shown in orange, the structural material in grey.

Our reference geometry is shown in Fig. 1, where, for generality, two piezoelectric layers are shown. To allow for both series an parallel connection of the bias voltage, the variable $b$ (with possible values \pm 1 ) is introduced. To understand the simplifications introduced by the various models, we need to introduce the most general constitutive equations for the piezoelectric material and the nonpiezoelectric structural material (Fig. 1). We write them in Voigt notation, using the uppercase $S$ and $P$ subscripts to indicate the material ( $S$ for structural, $P$ for piezo), and the lowercase subscripts for vector components (with values 1 , 2,3 corresponding to $x, y, z$ ), or tensor components in Voigt form (with the values $1,2,3,4,5,6$ corresponding to $x x$, $y y, z z, y z, x z, x y)$. With these conventions, and assuming summation over repeated indices, the equations read (IEEE
176-1987)

$$
\begin{aligned}
& S_{S, i}=s_{S, i j} T_{S, j}, \quad i, j=1 \ldots 6 \\
& S_{P, i}=s_{P, i j} T_{P, j}+d_{m i} E_{m}, \quad i, j=1 \ldots 6 ; m=1 \ldots 3 \\
& D_{m}=\epsilon_{m n} E_{n}+d_{m i} T_{P, i}, \quad i=1 \ldots 6 ; m, n=1 \ldots 3
\end{aligned}
$$

where $T_{*, j}$ indicates the stress tensor, $S_{*, i}$ indicates the strain tensor, $s_{*, i j}$ is the $6 \times 6$ compliance matrix (at constant electric field for the piezoelectric material), $E_{m}$ is the electric field, $D_{m}$ is the electric displacement, $\epsilon_{m n}$ is the $3 \times 3$ dielectric permittivity matrix (at constant stress) and $d_{m i}$ is the $6 \times 3$ piezoelectric strain matrix. For electrical and piezoelectrical quantities, which are only defined in the piezo, the material subscript is omitted.

We do not lose much generality if we assume hexagonal anisotropy for both materials. This type of anisotropy is typical of many piezoelectric materials such as PZT, AlN, $\mathrm{ZnO}$ (Auld 1975), and can also describe many structural materials such as aluminum, steel, single crystal silicon, polysilicon and others. We assume that the $z$ axis of the piezoelectric material is oriented parallel to the same axis of the system of coordinates in Fig. 1. This implies that the piezo is actuated in the $d_{31}$ mode, which is the most common in the literature. Alternative actuation modes, corresponding to the $d_{33}$ piezoelectric coefficients, are possible. The expanded matrix form of Eqs. (1a-1c) for hexagonal anisotropy is reported in Appendix A, where the generic compliance coefficients $s_{*, i j}$ are written as a function of the more commonly used Young's moduli $Y_{*, i}$, shear moduli $G_{*, i j}$ and Poisson's ratios $\nu_{*, i j}$. When the expanded notation is used, to simplify notation, we drop the subscripts for the elastic moduli referred to the $x$ and $y$ axes, i.e. we set $Y_{*, x}=Y_{*, y}=Y_{*}, G_{*, x y}=G_{*}$ and $\nu_{*, x y}=\nu_{*}$.

Essentially all existing models simplify Eq. (1c) by assuming that the in-plane components of the electric field and electric displacement are negligible:

$$
E_{1}=E_{2}=D_{1}=D_{2}=0 \text {. }
$$

Also, because it is generally assumed that the thicknesses are much smaller than the beam length $\left(t_{S}, t_{P} \ll L\right)$, the out-of-plane (i.e. along $z$ ) stress is always neglected as well:

$$
T_{S, 3}=T_{P, 3}=0
$$

The models can thus be classified depending on the assumptions made on the stress-strain state along the transverse $(y)$ direction. These assumptions depend on the beam geometry, whose defining quantities (the length $L$, width $W$, and piezo and structural thicknesses $t_{P}$ and $t_{S}$ ) are shown in Fig. 1 in the case of a triple layer bimorph. To simplify the classification, we define three adimensional 
parameters, the in-plane aspect ratio $\tilde{a}$, and the normalized piezo thickness $\tilde{t}$, and the slenderness $\tilde{s}$ :

$$
\tilde{a}=\frac{W}{L}, \quad \tilde{t}=\frac{t_{P}}{t_{S}}, \quad \tilde{s}=\frac{L}{t_{S}} .
$$

All three parameters span over more than two decades in actual devices [Fig. 2, (top)]. In the following, we will refer to three commonly used reduced models, i.e., the Null Transverse Stress (NTS) model, the Thin Film Continuous (TFC) model, and the Null Transverse Deformation (NTD) model.

The NTS model (Maurini et al. 2006; Gafforelli et al. 2015) is likely the most commonly used, and further assumes that:

$$
T_{S, 2}=T_{P, 2}=0 \text {. }
$$

Intuition suggests that this model is valid for narrow enough beams (i.e. for $\tilde{a} \ll 1$ ), under the argument that both the structural and piezoelectric materials can release their transverse stresses over the length of the beam. NTS was first derived by Crawley and De Luis (1987) and developed in several subsequent works (Gafforelli et al. 2015; Smits and Choi 1991; Smits et al. 1991; Smits and Ballato 1994; Qing-Ming Wang and Cross 1999; Weinberg 1999; Tadmor and Kosa 2003). Smits and coworkers derived the constitutive equations in matrix form for static cantilever unimorphs (Smits and Choi 1991) and static (Smits et al. 1991) as well as dynamic (Smits and Ballato 1994) cantilever bimorphs, while Wang and Cross considered the case of symmetric triple layer cantilevers (Qing-Ming Wang and Cross 1999). The same treatment was extended by Weinberg in order to take into account the effect of built-in axial stresses (Weinberg 1999), and by Tadmor and Kosa (2003), who considered the effect of bending strain on the electric field in the piezoelectric layers. Inman and coworkers developed a distributed parameter model of the beam frequency response (Erturk and Inman 2008), and used it to describe the experimental evidence obtained from several micro-engineered devices (Erturk and Inman 2009; Karami et al. 2011). Garcia and coworkers extended the theoretical model to the more general case of the TB model (Dietl et al. 2010), and also used it in experimental works on micro-engineered harvesters (Reissman et al. 2016). A similar study was also performed by Rafique and Bonello (2010). Interestingly, specific NTS assumptions on the geometry of the beam are frequently overlooked in the above literature and, when given, are not consistent. For example, Smits et al. (1991) mentions $\tilde{a} \ll 1$, while Smits and Ballato (1994); Qing-Ming Wang and Cross (1999) also require $W \gg t$; Weinberg (1999) requires $W \approx t$; Tadmor and Kosa (2003) requires $W<5 t$. Unfortunately, as no unified notation emerged in the above literature, a comparison of these models can be cumbersome and even challenging. To add another difficulty, some of these papers use a notation which may not be consistent with standard definitions (IEEE 176-1987), as we will detail below.

More troubling than notation issues is that NTS is often used when the piezoelectric layer is very thin (i.e. for $\tilde{t} \ll$ 1 ), when it is not realistic to assume that its stress state is not influenced by the (much thicker) structural layer. As a matter of fact, Eq. (5) leaves the layers free to deform independently along the $y$ axis, violating the continuity at their interface. This inconsistency is usually irrelevant in composite beams made only of structural elastic layers, as the difference in transverse deformation is only due to the different Poisson's ratios. In piezoelectric layers, instead, transverse deformations are also coupled to the electric field through the $d_{32}=d_{31}$ piezo-strain coefficient (see Appendix A), and this effect is relevant. To overcome this problem, Dubois and Muralt (1999) dropped Eq. (5) and assumed that a thin piezoelectric layer inherits the deformation of the structural layer, while the latter is still assumed to release its stress:

$$
S_{S, 2}=S_{P, 2}, \quad T_{S, 2}=0 .
$$

This model has been used in other works on the characterization of piezoelectric thin films (Defaÿ et al. 2006; Ledermann et al. 2003), as well as cases where a large difference between the thicknesses of the structural and piezoelectric layer exists. These include gas detectors exploiting gravimetric (Ivaldi et al. 2011) and photoacoustic (Ledermann et al. 2004) detection. We will refer to this model as the Thin Film Continuous (TFC) model.

Finally, for wide beams (i.e. when $\tilde{a} \geq 1$ ), which are often used in harvesting applications (Jackson et al. 2017; Olszewski et al. 2017; Jia and Seshia 2016; Lei et al. 2014) and accelerometers (Hindrichsen et al. 2010, 2009), the clamp at $x=0$ inhibits deformations along the entire length of the structure, so that negligible transverse deformation (or NTD) is often assumed:

$$
S_{S, 2}=S_{P, 2}=0
$$

NTD and NTS models are frequently presented together and labeled as plane strain and plane stress hypothesis, respectively (Weinberg 1999; Tadmor and Kosa 2003). Lei et al. (2014) used NTD assumptions to model their MEMS energy harvester. The same was done by Benjeddou et al. (1997) for their finite element model of piezoelectric beams and by Lim et al. (2001) in their theoretical study. NTD is also relevant in clamped-clamped beam resonators, as they are typically actuated and detected through piezoelectric patches placed at the clamps and covering only a small part of the total length of the beam (Karabacak et al. 2010; Piazza et al. 2004; DeVoe 2001; Torri et al. 2014) and, as such, the assumption of NTS (i.e. piezoelectric layers which are much longer than wide) is rarely true. Also in this case the literature gives inconsistent assumptions: Weinberg 
(1999) requires $W \gg t$, Tadmor and Kosa (2003) requires $W>5 t$, Lei et al. (2014) require $L, W \gg t$.

The intuitive geometrical conditions on $\tilde{a}$ and $\tilde{t}$ assumed for the three models are summarized in the second column of Table 2, and denoted by distinct color shades in Fig. 2 (top). However, the result of our Finite Element (FEM) simulations, summarized in Fig. 2 (bottom), show that these conditions are not always appropriate and, in the case of NTS and TFC, lead to very large errors. As a matter of fact, apart from the case of NTD, none of the conditions we summarized in the above discussion match the simulations results. Based on FEM results and physical insight, we developed revised geometrical conditions (Table 2, third column) that give a better description of the actual behavior. Their derivation will be detailed in the rest of the paper.

It is worth mentioning that more general models have been developed. For example, Dell'Isola and coworkers used a mixed variational approach to model narrow beams (Maurini et al. 2006). Their work can be seen as an attempt to unify the NTS and TFC models. Corigliano and coworkers (Gafforelli et al. 2015) developed a semianalytical expression based on the fitting of FEM results to describe the continuous variation from narrow to wide beam while keeping the thicknesses constant. The model can be thus seen as an attempt to unify NTS and NTD models.

The rest of the paper is organized as follows. In the next section, we develop a set of one-dimensional equivalent constitutive equations. Using a unified notation and equivalent mechanical and electrical parameters, we show that these equations have the same form for each of the three models. Assumptions and expected range of validity for each model are also examined. Then, the constitutive equations are used to develop a complete set of differential equations for the dynamical behavior of the beam. These equations are obtained by averaging the electrical and mechanical constitutive equations over a generic crosssection. We then propose and solve analytically a case study based on static loading of the piezoelectric composite beam. We then present FEM simulations of the same beam over a wide range of geometries and use the results to assess the range of validity of each model by comparison between FEM and theoretical expectations.

\section{Model}

Our derivation of the beam equations applies to generic beam structures and boundary conditions. However, for simplicity, we refer to the specific case of a piezoelectric triple layer cantilever bimorph as shown in Fig. 1, where the structural material is sandwiched between two identical piezoelectric layers. The clamped end is at $x=0$, while the other end (at $x=L$ ) is free. The origin of the reference system is placed at center of the beam cross section. We also assume that the top and bottom surfaces of the piezoelectric
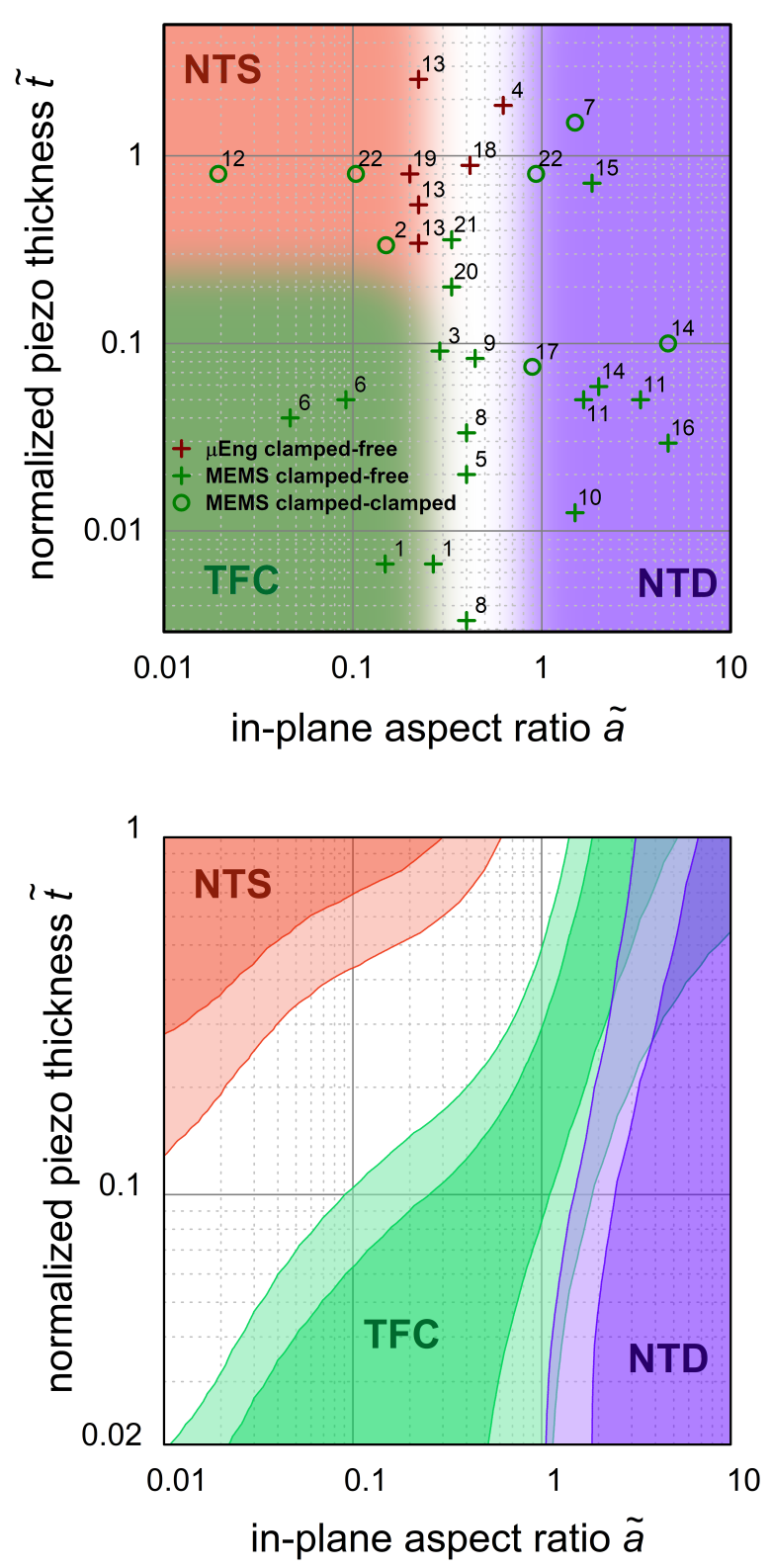

Figure 2. (top) In-plane aspect ratio $\tilde{a}$ and normalized piezo thickness $\tilde{t}$ for piezoelectric beams reported in literature, classified by boundary conditions and fabrication technology (microengineering or MEMS). The reference for each number is given in Table 1. For devices with piezoelectric patches, the in-plane aspect ratio of the patch is reported. The red, green, and purple areas give the expected ranges of validity for NTS, TFC and NTD, respectively. (bottom) For comparison, the actual ranges, as derived from FEM simulations, where the model errors are below 5\% (darker color) and 10\% (lighter color). 
Table 1. Correspondence table for reference numbers in Fig. 2 and Fig. 6.

\begin{tabular}{rl|rl} 
Ref. number & Reference & Ref. number & Reference \\
\hline 1 & Chen et al. (2006) & 12 & Karabacak et al. (2010) \\
2 & DeVoe (2001) & 13 & Karami et al. (2011) \\
3 & Dong et al. (2007) & 14 & Ledermann et al. (2004) \\
4 & Erturk and Inman (2009) & 15 & Lei et al. (2014) \\
5 & Glauvitz et al. (2012) & 16 & Olszewski et al. (2017) \\
6 & Hewa-Kasakarage et al. (2013) & 17 & Piazza et al. (2004) \\
7 & Hindrichsen et al. (2010) & 18 & Rafique and Bonello (2010) \\
8 & Isarakorn et al. (2011) & 19 & Reissman et al. (2016) \\
9 & Ivaldi et al. (2011) & 20 & Shibata et al. (2004) \\
10 & Jackson et al. (2017) & 21 & Shin et al. (2008) \\
11 & Jia and Seshia (2016) & 22 & Torri et al. (2014)
\end{tabular}

Table 2. Equivalent parameters for the reduced constitutive Eqs. (8) for the three different reduced models (NTS, NTD, TFC).

\begin{tabular}{|c|c|c|c|c|c|c|c|c|}
\hline model & $\begin{array}{c}\text { intuitive } \\
\text { geometric } \\
\text { conditions }\end{array}$ & $\begin{array}{c}\text { revised } \\
\text { geometric } \\
\text { conditions } \\
\text { (this work) }\end{array}$ & assumptions & $\begin{array}{c}K_{S} \\
\left(\mathrm{~N} / \mathrm{m}^{2}\right)\end{array}$ & $\begin{array}{c}K_{P} \\
\left(\mathrm{~N} / \mathrm{m}^{2}\right)\end{array}$ & $\begin{array}{c}\gamma_{m} \\
\left(\mathrm{C} / \mathrm{m}^{2}\right)\end{array}$ & $\begin{array}{c}\gamma_{e} \\
\left(\mathrm{C} / \mathrm{m}^{2}\right)\end{array}$ & $\begin{array}{c}\epsilon_{P} \\
(\mathrm{~F} / \mathrm{m})\end{array}$ \\
\hline NTS & $\tilde{a} \ll 1$ & $\begin{array}{c}\tilde{a} \ll 1, \\
\tilde{t} \approx 1 \text { or } \frac{\tilde{s}}{\tilde{s} \tilde{a}} \gtrsim 1\end{array}$ & $T_{S, 2}=T_{P, 2}=0$ & $Y_{S}$ & $Y_{P}$ & $d_{31} Y_{P}$ & $=\gamma_{m}$ & $\epsilon_{3}-d_{31}^{2} Y_{P}$ \\
\hline TFC & $\begin{array}{l}\tilde{a} \ll 1 \\
\tilde{t} \ll 1\end{array}$ & $\begin{array}{c}\tilde{a} \ll 1, \\
\tilde{t} \ll 1, \frac{\tilde{t}}{\tilde{s} \tilde{a}} \ll 1\end{array}$ & $\begin{array}{c}T_{S, 2}=0 \\
S_{S, 2}=S_{P, 2}\end{array}$ & $Y_{S}$ & $\frac{Y_{P}\left(1-\nu_{S} \nu_{P}\right)}{1-\nu_{P}^{2}}$ & $\frac{d_{31} Y_{P}}{1-\nu_{P}}$ & $\frac{d_{31} Y_{P}\left(1-\nu_{S}\right)}{1-\nu_{P}}$ & $\epsilon_{3}-\frac{2 d_{31}^{2} Y_{P}}{1-\nu_{P}}$ \\
\hline NTD & $\tilde{a} \geq 1$ & $\tilde{a} \geq 1$ & $S_{S, 2}=S_{P, 2}=0$ & $\frac{Y_{S}}{1-\nu_{S}^{2}}$ & $\frac{Y_{P}}{1-\nu_{P}^{2}}$ & $\frac{d_{31} Y_{P}}{1-\nu_{P}}$ & $=\gamma_{m}$ & $\epsilon_{3}-\frac{2 d_{31}^{2} Y_{P}}{1-\nu_{P}}$ \\
\hline
\end{tabular}

layers are equipotential, i.e. covered by a perfect electric conductor of negligible thickness, and that a voltage $V$ is applied to between top and bottom surfaces of the upper layer, and a voltage $b V$ is applied between the top and bottom surfaces of the lower layer, as illustrated in Fig. 1.

In the rest of this section we derive a single set of equations that are equivalent to any of the NTS, TFC, and NTD models, depending on the choice of a few parameters. The reader only interested in the results can jump directly to the presentation of the modified Euler-Bernoulli Model and refer, for the definition of the model parameters, to Table 2 and Table 4.

\section{Generalized Constitutive Equations}

Starting from Eqs. (29a)-(29c), we can solve for the axial stresses $T_{S, 1}, T_{P, 1}$ and the vertical electrical displacement $D_{3}$ by substituting Eqs. (2), (3) and one among Eqs. (5), (6) or (7). After a cumbersome but straightforward derivation, we can write their expressions as a function of five equivalent parameters with an intuitive physical meaning, whose values depend on the choice of the model:

$$
\begin{gathered}
T_{S, 1}=K_{S} S_{S, 1}, \\
T_{P, 1}=K_{P} S_{P, 1}-\gamma_{m} E_{3}, \\
D_{3}=\epsilon_{P} E_{3}+\gamma_{e} S_{P, 1} .
\end{gathered}
$$


$K_{S}, K_{P}$ are equivalent longitudinal stiffnesses associated with the beam and piezo respectively, $\gamma_{m}$ and $\gamma_{e}$ are equivalent coupling coefficients of mechanical and electrical equations respectively, and $\epsilon_{P}$ is an equivalent electrical permittivity. Their values for each of the three models are summarized in Table 2.

Table 2 shows that the three models (NTS, NTD, and TFC) can be unified under the same, compact constitutive Eqs. (8), with each model characterized by appropriate choice of the five parameters. The notation used in the several papers cited in the introduction is often different from the one adopted in this work. An attempt to organize and clarify these different notations is made in Appendix A, where alternative expressions for the equivalent parameters of Table 2 are also provided.

\section{Beam Equations}

Eqs. (8) are general relationships, only based on the assumptions of Eqs. 5-7. To determine the actual stressstrain field at each point in the beam, we now assume (as commonly done in the literature) that the strain distribution is that of the standard Euler-Bernoulli beam theory. Specifically, we write the axial strain $S_{*, 1}$ as the superposition of an extensional strain $\lambda(x)$ and a flexural deformation $\chi(x) \cdot z$

$$
S_{*, 1}(x, z)=\lambda(x)+\chi(x) z
$$

where $\lambda(x)$ and the curvature $\chi(x)$ are constant over the cross-section. The corresponding stress distributions in each layer will then be obtained by substituting Eq. 9 into Eqs. $8 \mathrm{a}-8 \mathrm{~b}$. Here, as in most literature, the dependence on $y$ is dropped (as in the standard Euler-Bernoulli model). An exception is (Gafforelli et al. 2015), where a specific functional dependence is initially retained and the stressstrain field is then averaged along $y$ with the aid of fitting parameters to reduce the dimensionality of the problem. Because of piezoelectric coupling, the cantilever may withstand extensional deformations (i.e. $\lambda(x)$ can be non-zero) even in absence of axial loads. Eqs. (8) and (9) can now be used to further reduce the dimensionality of the problem to one. Specifically, integration along $z$ of the axial stress $T_{*, 1}$ and electrical displacement $D_{3}$, taken from the constitutive equations at a generic cross-section, leads to a set of beam constitutive equations linking the charge per unit length $\rho(x)$, bending moment $M(x)$ and axial force $N(x)$ to the applied voltage $V$, curvature $\chi(x)$ and extensional deformation $\lambda(x)$. By substituting these constitutive equations into the differential equations of the Euler-Bernoulli model, the simplified 1D piezoelectric beam model is completed.

Charge per unit length. We derive the charge per unit length on the top/bottom surfaces of the piezoelectric

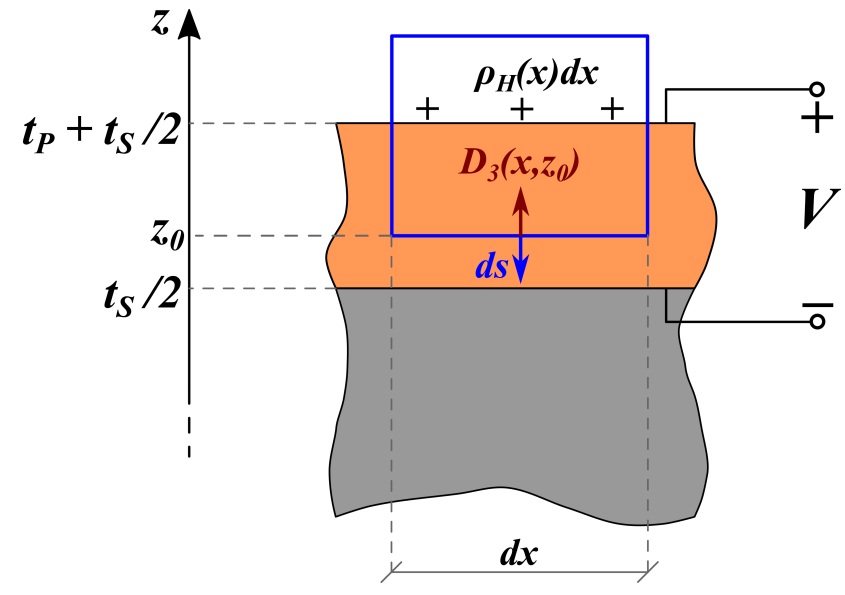

Figure 3. Gauss' theorem applied to the upper electrode of the top piezoelectric layer. The Gauss surface is represented in blue. The integration surface element has area $d s=W d x$.

layers by exploiting Gauss' flux theorem together with the constitutive electrical Eq. (8c). In this section we also show that, because of flexural deformations, the electric field inside the piezoelectric layers varies linearly across the thickness ( $z$ coordinate) with a slope proportional to the curvature $\chi(x)$. This effect is typically neglected (Erturk and Inman 2008; Dietl et al. 2010), as the common assumption is to consider the electric field independent of deformations. As will be shown in the following section, a non-constant electric field implies an additional, piezoelectrically-induced term in the beam bending stiffness. In the Case Study section we will discuss the range of validity of models neglecting this assumption. Among the papers cited in the introduction, the only ones considering the dependence of the electric field on the curvature are (Maurini et al. 2006; Tadmor and Kosa 2003; Benjeddou et al. 1997; Lim et al. 2001). The effect was also investigated by Krommer (Krommer 2001), Littrell and Grosh (Littrell and Grosh 2012) and Sze and coworkers (Sze et al. 2004).

To derive the charge expression, we first consider the top piezoelectric layer with the voltage $\mathrm{V}$ applied between its upper and lower surfaces. By applying the Gauss theorem to a surface enclosing a portion of length $\mathrm{dx}$ of the positive electrode (Fig. 3), we obtain

$$
-D_{3}\left(x, z_{0}\right) W d x=\rho_{H}(x) d x
$$

where $D_{3}\left(x, z_{0}\right)$ is the $z$ component of electric displacement inside the top piezoelectric layer and $\rho_{H}(x)$ is the charge per unit length on the surface of the positive electrode. Since $z_{0}$ is arbitrary, the electric displacement must be independent of $z$. The right-hand side of Eq. (8c) is therefore also independent of $z$. As $S_{P, 1}$, because of Eq. (9), 
is a linear function of $z$, so must be $E_{3}$. We then write

$$
E_{3}(x, z)=E_{3}^{(0)}(x)+E_{3}^{(1)}(x)\left(z-\frac{t_{P}+t_{S}}{2}\right)
$$

where $E_{3}^{(0)}$ (the field at the center of the layer) and $E_{3}^{(1)}$ (its slope) are suitable functions of $x$. Their values, as well as the ones relative to the bottom piezoelectric layer, can be easily determined by simple substitutions (see Appendix C). The complete expressions for electric fields $E_{3 H}, E_{3 L}$ inside the top and bottom piezoelectric layers, respectively, are (Tadmor and Kosa 2003; Benjeddou et al. 1997; Krommer 2001)

$$
\begin{aligned}
& E_{3 H}(x, z)=-\frac{V}{t_{P}}-\frac{\gamma_{e}}{\epsilon_{P}} \chi(x)\left(z-\frac{t_{P}+t_{S}}{2}\right) \\
& E_{3 L}(x, z)=-\frac{b V}{t_{P}}-\frac{\gamma_{e}}{\epsilon_{P}} \chi(x)\left(z+\frac{t_{P}+t_{S}}{2}\right) .
\end{aligned}
$$

The electric field expression has a first term which is constant and typical of parallel plate capacitors, and a second term proportional to the curvature and varying linearly with $z$. Neglecting the effect of deformations on the electric field means neglecting this latter term in Eqs. (12a)-(12b). Substituting Eqs. (12a)-(12b) and Eq. (9) into Eq. (8c) the expressions for the electric displacements are found

$$
\begin{gathered}
D_{3 H}(x)=-\epsilon_{P} \frac{V}{t_{P}}+\gamma_{e} \lambda(x)+\frac{1}{2} \gamma_{e}\left(t_{P}+t_{S}\right) \chi(x) \\
D_{3 L}(x)=-\epsilon_{P} \frac{b V}{t_{P}}+\gamma_{e} \lambda(x)-\frac{1}{2} \gamma_{e}\left(t_{P}+t_{S}\right) \chi(x) .
\end{gathered}
$$

Assuming a similar relationship for the bottom piezoelectric layer, Eqs. (13a)-(13b) can be finally used to compute the total charge per unit length through Eq. (10). Before this, however, we need to take into account the fact that $\rho_{H}$ is defined as the charge on the positive electrode (which, for the bottom piezoelectric layer, could be the lower one, depending on the sign of $b$ ), so that the sign of $D_{3 L}$ as presented in Eq. (13b) has to be reversed when $b=-1$, leading to

$$
\rho(x)=\rho_{H}(x)+\rho_{L}(x)=W\left(-D_{3 H}(x)-b D_{3 L}(x)\right) .
$$

By substituting Eqs. (13a)-(13b) we finally obtain

$$
\rho(x)=C_{l} V-\Gamma_{e L} \lambda(x)-\Gamma_{e F} \chi(x),
$$

where the expressions for the equivalent capacitance for unit length $C_{l}$, the electrical coupling coefficient for longitudinal deformations $\Gamma_{e L}$, and the electrical coupling coefficient for flexural deformations $\Gamma_{e F}$ are reported in Appendix B.
Bending Moment. The bending moment at a generic crosssection can be calculated by integrating the axial stresses multiplied by their respective arms on the cross-section surface

$$
\begin{aligned}
M(x)=W & \left(\int_{-\frac{t_{S}}{2}-t_{P}}^{-\frac{t_{S}}{2}} T_{P, 1}(x, z) z d z\right. \\
& +\int_{-\frac{t_{S}}{2}}^{\frac{t_{S}}{2}} T_{S, 1}(x, z) z d z \\
& \left.+\int_{\frac{t_{S}}{2}}^{\frac{t_{S}}{2}+t_{P}} T_{P, 1}(x, z) z d z\right) .
\end{aligned}
$$

By using the expressions of the axial stresses of Eqs. (8a), (8b) and substituting Eqs. (9) and (12a)-(12b), the previous equation reduces to

$$
M(x)=K I_{\text {tot }} \chi(x)+\Gamma_{m F} V,
$$

where the expressions of the total flexural stiffness $K I_{t o t}$ and the mechanical coupling coefficient for flexural deformations $\Gamma_{m F}$ are reported in Appendix B. The total flexural stiffness $K I_{t o t}$ is the sum of the flexural stiffness of the composite beam $K I$, plus a piezoelectrically-induced flexural stiffening introduced by the curvature-dependent term of the electric field. This latter term vanishes if the electric field is assumed constant. The expression of the flexural stiffness of the composite beam $K I$ is given by the standard theory of composite beams (Lobontiu and Garcia 2005) using the stiffnesses $K_{S}, K_{P}$ of Table 2 in place of the Young's moduli.

Axial force. The approach used to compute the moment can be repeated to determine the axial force:

$$
\begin{aligned}
N(x)=W & \left(\int_{-\frac{t_{S}}{2}-t_{P}}^{-\frac{t_{S}}{2}} T_{P, 1}(x, z) d z\right. \\
& +\int_{-\frac{t_{S}}{2}}^{\frac{t_{S}}{2}} T_{S, 1}(x, z) d z \\
& \left.+\int_{\frac{t_{S}}{2}}^{\frac{t_{S}}{2}+t_{P}} T_{P, 1}(x, z) d z\right),
\end{aligned}
$$

and, with the same substitutions as above, we obtain

$$
N(x)=K A \lambda(x)+\Gamma_{m L} V
$$

where the expressions of the longitudinal stiffness $K A$ and mechanical coupling coefficient for longitudinal deformations $\Gamma_{m L}$ are again reported in Appendix B. The expression of the longitudinal stiffness $K A$ is given by the standard theory of composite rods (Lobontiu and Garcia 2005 ) with again the equivalent stiffnesses $K_{S}, K_{P}$ in place of the Young's moduli. 
Matrix form Eqs. (15), (17) and (19) can be collected in matrix form:

$$
\left[\begin{array}{c}
N(x) \\
M(x) \\
\rho(x)
\end{array}\right]=\left[\begin{array}{ccc}
K A & 0 & \Gamma_{m L} \\
0 & K I_{t o t} & \Gamma_{m F} \\
-\Gamma_{e L} & -\Gamma_{e F} & C_{l}
\end{array}\right]\left[\begin{array}{c}
\lambda(x) \\
\chi(x) \\
V
\end{array}\right] .
$$

The matrix is, in general, not antisymmetric. However:

- for the NTD and NTS models, $\gamma_{m}=\gamma_{e}$ (See Table 2), the mechanical and electrical coupling coefficients are identical (see Table 4), and the matrix is antisymmetric;

- if the piezoelectric layers are biased with the same orientation (i.e. $b=1$ ), the flexural coupling cofficients $\Gamma_{m F}$ and $\Gamma_{e F}$ vanish, and the voltage is coupled to longitudinal deformations only;

- if the bias orientation is alternate $(b=-1)$, the longitudinal coupling coefficients $\Gamma_{m L}$ and $\Gamma_{e L}$ vanish, and the voltage is coupled to flexural deformations only.

Typical polarization schemes exploit parallel and series connections, as well as the different poling of the piezoelectric layers (Smits et al. 1991; Qing-Ming Wang and Cross 1999). The symmetric triple layer bimorph show in Fig. 1 is typically polarized for flexural deformations. For unimorph beams, longitudinal and flexural deformations are intrinsically coupled (Luschi and Pieri 2015). The expressions of the coefficients appearing in Eq. (20) are summarized in Appendix B, where the expressions for unimorph beams are reported as well.

\section{Modified Euler-Bernoulli Model for Piezoelectric Beam}

We are now ready to generalize the differential equations governing flexural and longitudinal deformations of beams to include the effect of piezoelectric coupling described by Eq. (20). Flexural deformations can be studied by means of the Euler-Bernoulli model (Hagedorn and DasGupta 2007). Following the approach in (Reissman et al. 2016), (Luschi et al. 2016), we write them in matrix form. This is done by substituting the expression of the bending moment $M(x)$ of Eq. (20) into the matrix form of Euler-Bernoulli equation, to obtain:

$$
\begin{aligned}
\frac{\partial}{\partial x}\left[\begin{array}{c}
U_{z}(x, t) \\
\Psi(x, t) \\
M(x, t) \\
T(x, t)
\end{array}\right]= & {\left[\begin{array}{cccc}
0 & 1 & 0 & 0 \\
0 & 0 & -1 / K I_{t o t} & 0 \\
0 & 0 & 0 & 1 \\
0 & 0 & 0 & 0
\end{array}\right]\left[\begin{array}{c}
U_{z}(x, t) \\
\Psi(x, t) \\
M(x, t) \\
T(x, t)
\end{array}\right] } \\
& +\left[\begin{array}{c}
V(t) \Gamma_{m F} / K I_{\text {tot }} \\
0 \\
-p(x, t)+\mu_{l} \ddot{U}_{z}(x, t)
\end{array}\right]
\end{aligned}
$$

where $U_{z}(x, t)$ and $\Psi(x, t)$ are the out-of-plane deflection and rotation angle of the cross-section, respectively, $T(x, t)$ is the shear force at the cross-section, $p(x, t)$ is the distributed load per unit length along the beam and $\mu_{l}$ is the mass per unit length. Straightforward expressions for the mass per unit length for unimorph and bimorph beams are given in Appendix B. In writing Eq. (21), the relationship between rotation angle and curvature $\chi(x, t)=$ $-\partial \Psi(x, t) / \partial x$ has been used, and dots have been used to indicate time derivatives.

A corresponding derivation can be performed for longitudinal deformations (Hagedorn and DasGupta 2007). By substituting the expression of the axial force $N(x, t)$ obtained from the beam constitutive Eq. (20), the matrix form of the differential system becomes

$$
\begin{aligned}
\frac{\partial}{\partial x}\left[\begin{array}{c}
U_{x}(x, t) \\
N(x, t)
\end{array}\right]= & {\left[\begin{array}{cc}
0 & 1 / K A \\
0 & 0
\end{array}\right]\left[\begin{array}{c}
U_{x}(x, t) \\
N(x, t)
\end{array}\right] } \\
& +\left[\begin{array}{c}
-\Gamma_{m L} K A V(t) \\
-q(x, t)+\mu_{l} \ddot{U}_{x}(x, t)
\end{array}\right]
\end{aligned}
$$

where $U_{x}(x, t)$ and $q(x, t)$ are the axial displacement of the cross-section and the distributed axial load per unit length, respectively. In writing Eq. (22) we used the relationship between the longitudinal strain and axial displacement $\lambda(x, t)=\partial U_{x}(x, t) / \partial x$. One last equation for the electrical port is needed to completely describe the system. This is obtained by integrating $\rho(x, t)$ over the length of the cantilever

$$
\begin{aligned}
Q(t)= & -\Gamma_{e L}\left[U_{x}(L, t)-U_{x}(0, t)\right] \\
& +\Gamma_{e F}[\Psi(L, t)-\Psi(0, t)]+C_{0} V(t)
\end{aligned}
$$

where $Q(t)$ is the total charge on the electrodes and $C_{0}=$ $C_{l} L$ the total capacitance. Moreover, if, for example, the electric port is closed on a resistive load $R$ (a configuration typically used to evaluate the performances of piezoelectric energy harvesters (Erturk and Inman 2009; Karami et al. 2011; Reissman et al. 2016; Rafique and Bonello 2010)), the additional equation

$$
\frac{d}{d t} Q(t)=-\frac{V(t)}{R} .
$$

is required. The method of the transmission matrices can be used as an efficient solution technique for this system of differential Eqs. (21-24) (Reissman et al. 2016; Luschi and Pieri 2015; Luschi et al. 2016).

\section{Case Study}

As a case study we consider the triple layer cantilever bimorph of Fig. 1 with flexural coupled polarization $(b=$ -1 ). We apply a static moment (per unit width) $M_{L}$ at the free end and determine the open circuit voltage $V_{o c}$ 
(Fig. 4, inset). This configuration is common in harvesting applications (Erturk and Inman 2009; Rafique and Bonello 2010; Maurini et al. 2006; Dietl et al. 2010). Since there are no axial loads, and $\Gamma_{e L}, \Gamma_{m L}$ are zero due to the chosen bias, the system is fully described by Eqs. (21) and (23). By solving with the boundary conditions $U_{z}(0)=$ $\Psi(0)=T(L)=Q=0$ and $M(L)=M_{L} W$, the following expression for the open circuit voltage is found

$$
V_{o c}=\frac{\Gamma_{e F} L}{C_{0} K I_{t o t}+\Gamma_{e F} \Gamma_{m F} L} M_{L} W .
$$

It is useful to express the open circuit voltage as a function of the equivalent constitutive parameters and the geometrical adimensional coefficients $\tilde{t}$ and $\tilde{a}$, substituting the expressions of Appendix B:

$$
V_{o c}=\frac{\gamma_{e}(1+\tilde{t}) 6 \tilde{t}}{\left(\begin{array}{c}
\epsilon_{P}\left[K_{S}+K_{P}\left(6 \tilde{t}+12 \tilde{t}^{2}+8 \tilde{t}^{3}\right)\right]+ \\
2 \gamma_{e} \gamma_{m} \tilde{t}^{3}+6 \gamma_{e} \gamma_{m} \tilde{t}(1+\tilde{t})^{2}
\end{array}\right)} \frac{1}{t_{S}} M_{L}
$$

The five equivalent parameters $K_{S}, K_{P}, \gamma_{m}, \gamma_{e}$ and $\epsilon_{P}$ have different expressions for the NTS, TFC and NTD models (Table 2). These parameters impose an additional implicit dependence of the voltage on $\tilde{t}$ and $\tilde{a}$. In the following we will investigate this dependence and verify the predictions of the three models by comparing Eq. (26) with FEM simulations performed over a wide range of geometries. FEM simulations were performed with ANSYS, using SOLID186 and SOLID226 elements to mesh the volumes associated with the structure and piezoelectric material, respectively. The number of elements along the thicknesses was kept constant at 2 and 1 for the structural and piezoelectric material, respectively, while the number of in plane (i.e. along $W$ and $L$ ) elements are varied with the structure geometry. The applied moment per unit width is set to $M_{L}=-10^{-3} \mathrm{~N}$. The simulated structural material is polysilicon, while the piezoelectric layers are made of PZT-5H. Material properties, taken from (Cho and Chasiotis 2007) and (Auld 1975), respectively, are reported in Table 3.

We simulate several different geometries by sweeping $\tilde{a}$ between 0.01 and $10, \tilde{t}$ between 0.02 and 1 , and $\tilde{s}$ between 30 and 150 while keeping the structural thickness $t_{S}$ constant at $6 \mu \mathrm{m}$. These ranges include most real geometries in the literature (see Fig. 2).

We first analyze the impact of the (normalized) piezoelectric thickness $\tilde{t}$, comparing the predictions of the analytical model of Eq. (26) and FEM results. In Fig. 4, the voltage $V_{o c}$ as a function of $\tilde{t}$ for the three analytical models and for two FEM cases, corresponding to the lower and upper bounds of $\tilde{a}$, are given (the slenderness $\tilde{s}$ is kept constant at 30 in both cases). The voltage has a maximum for a specific piezo thickness, a behavior has already been documented in the literature (Karami et al. 2011). In agreement with our previous discussion on the range of validity of the models, for narrow beams $(\tilde{a}=0.01)$ the FEM data moves from the TFC to the NTS model as $\tilde{t}$ increases. The agreement is confirmed for wide beams $(\tilde{a}=10)$, where the behavior is completely caught by the NTD model.

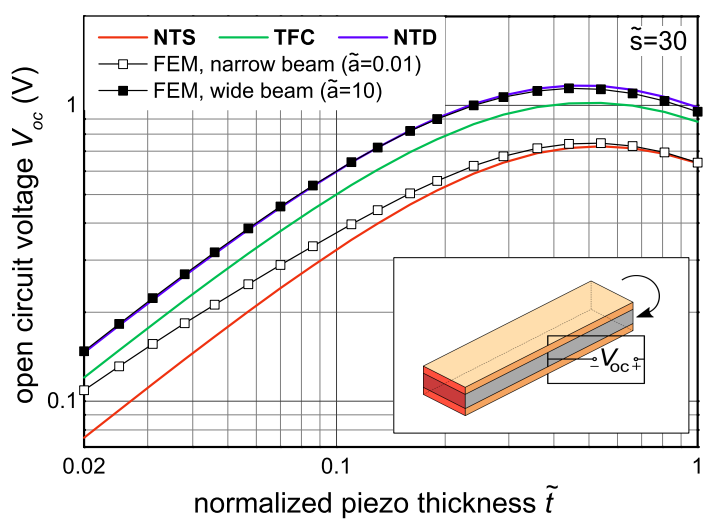

Figure 4. FEM open circuit voltage as a function of $\tilde{t}$ for analytical and FEM models; the voltage polarity and loading torque direction are shown in the inset.

Other aspects can be appreciated by looking at the plots of Fig. 5, where we give the voltage $V_{o c}$ as a function of $\tilde{a}$ for the three analytical models and for two FEM cases, corresponding to the lower and upper bounds of $\tilde{t}$ (the slenderness $\tilde{s}$ is kept constant at 30 in both cases). Since no explicit dependence on $\tilde{a}$ is present in the models, they appear as horizontal lines in these plots. The monotone behavior of the FEM voltage with respect to $\tilde{a}$ is also documented in (Gafforelli et al. 2015) and has its upper limit well predicted by the NTD model for both thin (lower graph, low $\tilde{t}$ ) and thick (higher graph, high $\tilde{t}$ ) piezoelectric layers. The lower limit is instead predicted by the TFC and NTS models for thin and thick piezo layers, respectively. Once again, such behavior is in agreement with our discussion on the range of validity of the models. In these plots NTS and NTD models are also reported (as dashed lines) assuming a constant electric field and thus disregarding the piezoelectrically induced flexural stiffening in $K I_{\text {tot }}$ (see Table 3 ). The data reveals that this effect gives a noticeable improvement to the NTD model only for thick piezoelectric layers.

A comprehensive assessment of the range of validity of the models can be obtained by defining the relative error of each model as

$$
E_{r}=\frac{V_{o c}(\text { model })-V_{o c}(\mathrm{FEM})}{V_{o c}(\mathrm{FEM})}
$$


Table 3. Material properties used in the example. Symbols are defined in Appendix A.

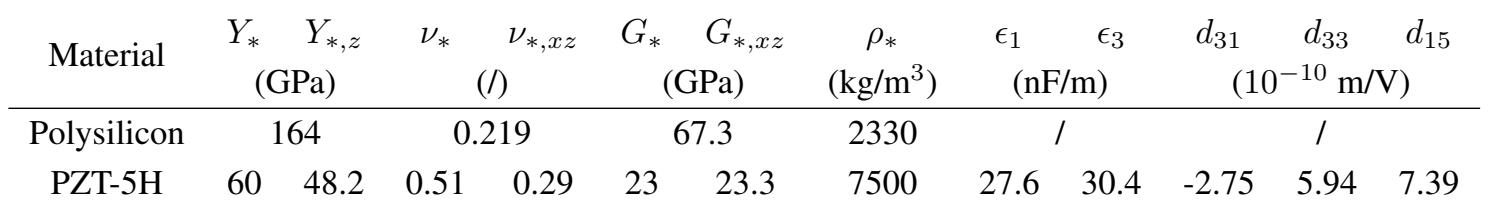

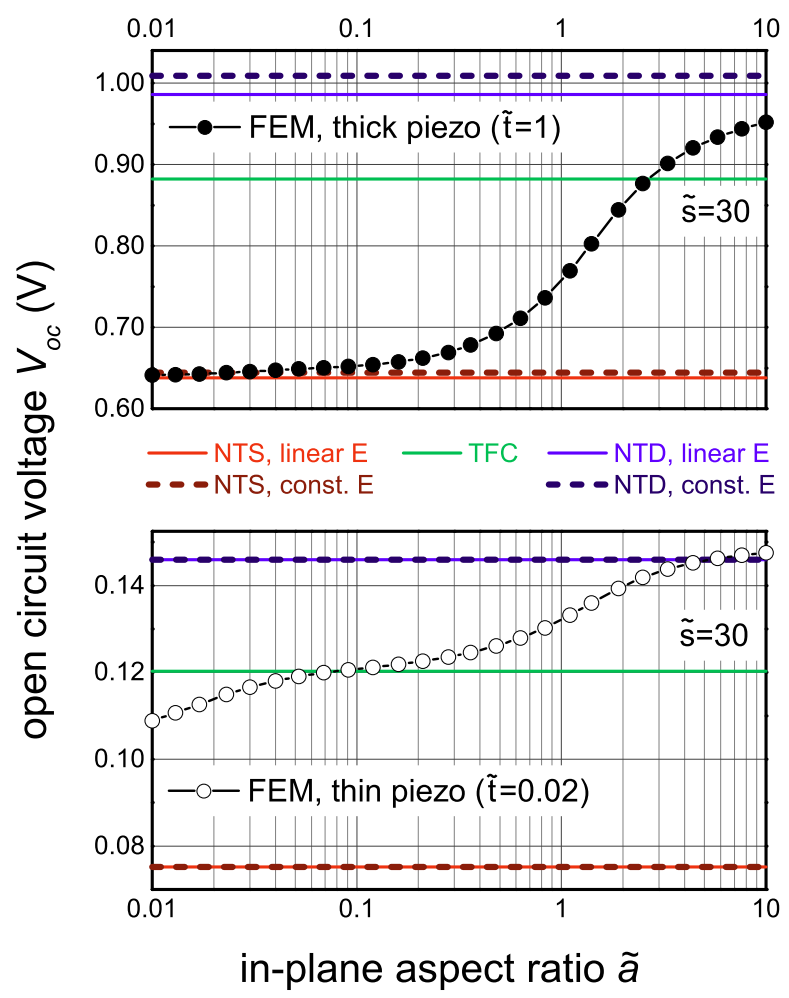

Figure 5. Open circuit voltage as a function of $\tilde{a}$ for the upper and lower bounds of the normalized piezo thickness, $\tilde{t}=1$ (top) and $\tilde{t}=0.02$ (bottom).

and plot them in the $[\tilde{a}, \tilde{t}]$ design space. A plot of the absolute value of the relative error is given in Fig. 6 for three values of the slenderness $\tilde{s}$. The stars in Fig. 6 represent actual devices (reported in the references) for which the authors have presented an analytical model, with the star color referring to the used model (red for NTS, green for TFC, purple for NTD). Each star has been placed in the plot with the value of $\tilde{s}$ closest to the real one. Comparison of solid and dashed lines in Fig. 6 shows that the assumption of field-induced flexural stiffness is only significant for the NTD model. This confirms the behavior shown in Fig. 5 for thick piezoelectric layers.

The data in Fig. 6 also clearly shows that there is a large domain of geometries, where real devices commonly lie, for which none of the usually used models gives accurate predictions. Most importantly, it shows that NTS is frequently used far outside of its reasonable range of validity. Moreover, sometimes, as in (Ledermann et al. 2004), TFC has been used when NTD would be more accurate.

Using the error zones of Fig. 6, a critical review of the ranges of validity discussed in the initial section and given in the second column of Table 2 for each of the three analytical models is possible. The condition on NTD (i.e. $\tilde{a} \geq 1$ ) is essentially respected, but the conditions for the NTS model $(\tilde{a} \ll 1)$ and the TFC model $(\tilde{a} \ll 1$ and $\tilde{t} \ll 1$ ) do not capture the FEM behavior. The transition between NTS and TFC is ruled by both $\tilde{a}$ and $\tilde{t}$, and is also somewhat dependent on the slenderness, with the NTS model more accurate for a smaller slenderness.

A possible explanation for this effect can be given by reconsidering the argument behind the choice of the NTS model, that is, the assumption that, for sufficiently long beams, the piezo can release its transverse stress because it is not constrained significantly by the clamp. This, of course, might not be the case if the piezo is so thin that its deformation is imposed by the underlying structural layer (hence the need, correctly identified by some authors, of the TFC model). Fig. 6 shows that a beam with a thin piezo $(\tilde{t} \ll 1)$ can nevertheless obey NTS, if the beam is narrow enough. This behavior can be explained by noting that, even if the piezo is thin with respect to the beam, it would be free to expand along $y$ (and thus release the transverse stress) as long as it is narrow, i.e. for $t_{P} \gtrsim W$. This inequality can be translated in adimensional form as:

$$
\frac{\tilde{t}}{\tilde{a} \tilde{s}} \gtrsim 1 .
$$

It is thus reasonable that NTS retains its validity even at small $\tilde{t}$ 's, if Eq. (28) holds, and also takes into account the dependence on the slenderness of the NTS validity domain. Conversely, the assumption at the base of TFC, i.e. the continuity of transverse deformation of the two layers, does not seem justified for this kind of geometries, even for small $\tilde{t}$ 's.

Figure 6 also shows, however, that for $t_{P} \approx t_{S}$ and moderate beam widths ( $\tilde{a}$ between 0.1 and 1), i.e. in the upper central portion of the graphs, the validity of NTS extends to the right of the domain defined by Eq. (28). A possible explanation is related to the fact that the piezolectrically-induced transverse stress is distributed along the whole thickness $t_{P}$ of the piezo. A larger 



Figure 6. Regions in the $[\tilde{a}, \tilde{t}]$ space where the analytical models provide an estimate of the open-circuit voltage $V_{o c}$ with $\left|E_{r}\right|$ less than $5 \%$ (darker color) and $10 \%$ (lighter color) at three different values of $\tilde{s}$. Data in the shaded area are interpolated. Dashed lines assume a constant electric field, solid lines a non-constant one. Actual structures from the literature are marked with a star and color-coded depending on the model used in the reference. The reference for each number is given in Table 1.

thickness may then favor the release of this stress in that part of the piezo which is further away from the interface between the two layers, thus causing an extension of the NTS range even at larger widths. Consequently, we propose that NTS is accurate when $\tilde{a} \ll 1$ and either $\tilde{t} / \tilde{a} \tilde{s} \gtrsim 1$ or $\tilde{t} \approx 1$ is true (Table 2 ).

Finally, from Fig. 6 appears that TFC behaves as an intermediate model between NTS and NTD over the whole design domain, even for large $\tilde{t}$ 's, where, strictly based on its assumptions, it should not be applied. This fact is confirmed also by Figs. 4-5, where the green TFC curve always lies between the other two, and is likely a consequence of the transitional nature of the model.

\section{Conclusions}

It this work, we critically review the most commonly used simplified models for the electromechanical behavior of composite piezoelectric beams. These models essentially differ on the assumptions used to simplify the complex stress-strain state at the beam cross-section and reduce the dimensionality of the problem. We develop a compact formalism which incorporates three different models into a single, generalized set of equations. The parameters of this model can be chosen depending on the validity of certain assumptions, which in turn depend on the beam geometry.
The model can be used both for unimorph and bimorph beams.

We validate our model with extensive FEM simulations of a piezoelectric bimorph beam for hundreds of different geometries, showing that the models broadly used in the literature are often applied in a way that can lead to very large errors, even when the geometric conditions of validity commonly assumed for these models are satisfied. We then propose revised geometric conditions that match the expected behavior more accurately.

Our approach presents two distinct advantages for designers: first, they are given a single, unified equation which can be used to obtain accurate predictions for any geometry of the beam; moreover, the dependence on the beam geometry is included through the use of equivalent parameters with clear physical meaning, and these parameters can be easily computed based on simple selection rules which, in turn, depend on the beam geometry. We expect our model and geometric criteria for model selection to become a valuable help for the designer of piezoelectric sensors and actuators in a variety of applications.

\section{Appendix A}

Assuming hexagonal anisotropy constitutive Eqs. (1) can be written in matrix form as 


$$
\begin{aligned}
& {\left[\begin{array}{l}
S_{P, 1} \\
S_{P, 2} \\
S_{P, 3} \\
S_{P, 4} \\
S_{P, 5} \\
S_{P, 6}
\end{array}\right]=\left[\begin{array}{cccccc}
\frac{1}{Y_{P}} & -\frac{\nu_{P}}{Y_{P}} & -\frac{\nu_{P, x z}}{Y_{P}} & 0 & 0 & 0 \\
-\frac{\nu_{P}}{Y_{P}} & \frac{1}{Y_{P}} & -\frac{\nu_{P, x z}}{Y_{P}} & 0 & 0 & 0 \\
-\frac{\nu_{P, x z}}{Y_{P}} & -\frac{\nu_{P, x z}}{Y_{P}} & \frac{1}{Y_{P, z}} & 0 & 0 & 0 \\
0 & 0 & 0 & \frac{1}{G_{P, x z}} & 0 & 0 \\
0 & 0 & 0 & 0 & \frac{1}{G_{P, x z}} & 0 \\
0 & 0 & 0 & 0 & 0 & \frac{1}{G_{P}}
\end{array}\right]\left[\begin{array}{c}
T_{P, 1} \\
T_{P, 2} \\
T_{P, 3} \\
T_{P, 4} \\
T_{P, 5} \\
T_{P, 6}
\end{array}\right]+\left[\begin{array}{ccc}
0 & 0 & d_{31} \\
0 & 0 & d_{31} \\
0 & 0 & d_{33} \\
0 & d_{15} & 0 \\
d_{15} & 0 & 0 \\
0 & 0 & 0
\end{array}\right]\left[\begin{array}{c}
0 \\
0 \\
E_{3}
\end{array}\right]} \\
& {\left[\begin{array}{c}
D_{1} \\
D_{2} \\
D_{3}
\end{array}\right]=\left[\begin{array}{ccc}
\epsilon_{1} & 0 & 0 \\
0 & \epsilon_{1} & 0 \\
0 & 0 & \epsilon_{3}
\end{array}\right]\left[\begin{array}{c}
0 \\
0 \\
E_{3}
\end{array}\right]+\left[\begin{array}{cccccc}
0 & 0 & 0 & 0 & d_{15} & 0 \\
0 & 0 & 0 & d_{15} & 0 & 0 \\
d_{31} & d_{31} & d_{33} & 0 & 0 & 0
\end{array}\right]\left[\begin{array}{l}
T_{P, 1} \\
T_{P, 2} \\
T_{P, 3} \\
T_{P, 4} \\
T_{P, 5} \\
T_{P, 6}
\end{array}\right] .} \\
& d_{15}=\frac{e_{15}}{G_{P, x z}} .
\end{aligned}
$$
equations (8) of NTS, TFC and NTD models are found. The expressions of the five equivalent parameters appearing in such equations are reported in Table 2.

Many references use different and sometimes misleading notations for these same coefficients. It is common to NTS models (see for example (Karami et al. 2011; Dietl et al. 2010; Torri et al. 2014)) to call the equivalent coupling coefficients $\gamma_{m}=\gamma_{e}$ with the symbol $e_{31}$, and the equivalent dielectric permittivity $\epsilon_{P}$ with the symbol $\epsilon_{3}^{S}$. This notation can be confusing, as these symbols are typically used to identify an element of the piezoelectric stress matrix $\left(e_{31}\right)$ and of the dielectric permittivity tensor as constant strain $\left(\epsilon_{3}^{S}\right)$, respectively (IEEE 176-1987), and, in general, there is no identity between the two. Moreover, some references (see for example (Dietl et al. 2010)), use the symbol $C_{11}$ for the Young's modulus, which is the standard symbol for the 11 element of the stiffness matrix (at constant electric field) $c_{*, i j}=\left(s_{*, i j}\right)^{-1}$ and is, in general, different from the Young's modulus.

For further clarity, we also report below the relationships among these coefficients assuming hexagonal anisotropy. The piezoelectric stress matrix $e_{m i}$ has the same form of the piezoelectric stain matrix $d_{m i}$ reported in Eq. (29c) and can be written as $e_{m i}=d_{m k} c_{P, k i}$. The extended expressions relating the coefficients of the two piezoelectric matrices are

$$
\begin{gathered}
e_{31}=\frac{Y_{P}\left(Y_{P} d_{31}+d_{33} Y_{P, z} \nu_{P, x z}\right)}{Y_{P}\left(1-\nu_{P}\right)-2 Y_{P, z} \nu_{P, x z}^{2}}, \\
e_{33}=\frac{Y_{P} Y_{P, z}\left(d_{33}\left(1-\nu_{P}\right)+2 d_{31} \nu_{P, x z}\right)}{Y_{P}\left(1-\nu_{P}\right)-2 Y_{P, z} \nu_{P, x z}^{2}}, \\
e_{15}=d_{15} G_{P, x z} \\
d_{31}=\frac{e_{31}\left(1-\nu_{P}\right)-e_{33} \nu_{P, x z}}{Y_{P}} \\
d_{33}=\frac{e_{33}}{Y_{P, z}}-\frac{2 e_{31} \nu_{P, x z}}{Y_{P}}
\end{gathered}
$$

The dielectric permittivity matrix at constant strain $\epsilon_{m n}^{S}$ has the same form of the permittivity matrix at constant stress $\epsilon_{m n}$ reported in Eq. (29c) and can be written as $\epsilon_{m n}^{S}=\epsilon_{m n}-d_{m k} e_{k n}$. The extended expression relating the coefficients of the two permittivity matrices are

$$
\epsilon_{1}^{S}=\epsilon_{1}-d_{15} e_{15}, \quad \epsilon_{3}^{S}=\epsilon_{3}-2 d_{13} e_{13}-d_{33} e_{33} .
$$

We note here that the equalities $e_{31}=d_{31} Y_{P}$ and $\epsilon_{3}^{S}=$ $\epsilon_{3}-d_{31}^{2} Y_{P}$ suggested by the notations used in (Karami et al. 2011; Dietl et al. 2010; Torri et al. 2014) are true if and only if a simplified version of the piezoelectric stress matrix, where only the 31 element $e_{31}$ is nonzero, is assumed, a condition never verified in real piezoelectric materials.

A further useful clarification on notation is required for many works regarding TFC and NTD models (see for example (Defaÿ et al. 2006; Ledermann et al. 2003; Benjeddou et al. 1997)). In these works, a notation exploiting the piezoelectric stress coefficients, as well as the stiffness coefficients is used. By means of simple substitutions it is easy to verify the identities among such expressions and the ones used in this work:

$$
\begin{gathered}
K_{*} \stackrel{\text { NTD }}{\longrightarrow} \frac{Y_{*}}{1-\nu_{*}^{2}}=c_{*, 11}-\frac{c_{*, 13}^{2}}{c_{*, 33}} \\
\gamma_{m} \stackrel{\text { TFC,NTD }}{\longrightarrow} \frac{d_{31} Y_{P}}{1-\nu_{P}}=e_{31}-\frac{c_{P, 13}}{c_{P, 33}} e_{33} \\
\epsilon_{P} \stackrel{\text { TFC,NTD }}{\longrightarrow} \epsilon_{3}-\frac{2 d_{31}^{2} Y_{P}}{1-\nu_{P}}=\epsilon_{3}^{S}+\frac{e_{33}^{2}}{c_{P, 33}}
\end{gathered}
$$

\section{Appendix B}

The expressions of the parameters appearing in the differential system (21-23) are reported in Table 4 for symmetric triple layer bimorphs and unimorphs piezoelectric beams. The triple layer bimorph geometry of 
Fig. 1 has been introduced and taken as a case study for the derivation of such parameters in the Model section. The results are summarized here for convenience. By following the same procedure with minimal changes, the expressions for the unimorph geometry can be obtained. For this case, only the top piezoelectric layer is retained.

In the same table, the position $z_{N}$ of the neutral axis (computed from the bottom of the stack) and the expression of the mass per unit length $\mu_{l}$, are also reported. In the expression of $\mu_{l}$ the symbols $\rho_{S}$ and $\rho_{P}$ represent the mass density of the structural and piezoelectric material, respectively.

The position of the neutral axis is straightforward for the triple layer bimorph due to the symmetry of the system. For a unimorph geometry the position of the neutral axis involves basic beam computations (Lobontiu and Garcia 2005), once again using the equivalent longitudinal stiffnesses $K_{S}, K_{P}$ in place of the Young's moduli.

While computing the parameters in Table 4, we choose to place the origin of the reference system at the neutral axis. It is worth noting that this choice is arbitrary but any choice different from the one adopted here has the significant drawback of leading to a beam constitutive matrix of Eq. (20) with nonzero elements at positions 12 and 21, as suggested in (Littrell and Grosh 2012).

\section{Appendix C}

The complete expression of the electric field inside the top piezoelectric layer can be determined by substituting Eqs. (9) and (11) into Eq. (8c)

$$
\begin{aligned}
D_{3}(x)= & \epsilon_{P} E_{3}(x, z)+\gamma_{e} S_{P, 1}(x, z) \\
= & \epsilon_{P}\left[E_{3}^{(0)}(x)+E_{3}^{(1)}(x)\left(z-\frac{t_{P}+t_{S}}{2}\right)\right] \\
& +\gamma_{e}[\lambda(x)+\chi(x) z] \\
= & \epsilon_{P}\left[E_{3}^{(0)}(x)-E_{3}^{(0)}(x) \frac{t_{P}+t_{S}}{2}\right] \\
& +\gamma_{e} \lambda(x)+z\left[\epsilon_{P} E_{3}^{(1)}(x)+\gamma_{e} \chi(x)\right] .
\end{aligned}
$$

The independence on $z$ of the electric displacement $D_{3}(x)$ requires that the last term vanishes and, consequently

$$
E_{3}^{(1)}(x)=-\frac{\gamma_{e}}{\epsilon_{P}} \chi(x) .
$$

$E_{3}^{(0)}(x)$ can be obtained by integration of Eq. (11) along the layer thickness

$$
\begin{gathered}
\int_{\frac{t_{S}}{2}}^{\frac{t_{S}}{2}+t_{P}} E_{3}(x, z) d z=E_{3}^{(0)}(x) t_{P}=-V \\
E_{3}^{(0)}(x)=-\frac{V}{t_{P}} .
\end{gathered}
$$

By substituting Eqs. (34) and (35b) into Eq. (11) the complete expression of the electric field is found.

\section{References}

Auld BA (1975) Acoustic fields and waves in solids, volume I. DOI:10.1016/0003-682X(75)90008-0.

Benjeddou A, Trindade MA and Ohayon R (1997) A Unified Beam Finite Element Model for Extension and Shear Piezoelectric Actuation Mechanisms. Journal of Intelligent Material Systems and Structures 8(12): 1012-1025. DOI: 10.1177/1045389X9700801202.

Chen SN, Wang GJ and Chien MC (2006) Analytical modeling of piezoelectric vibration-induced micro power generator. Mechatronics 16(7): 379-387. DOI: 10.1016/j.mechatronics.2006.03.003.

Cho SW and Chasiotis I (2007) Elastic Properties and Representative Volume Element of Polycrystalline Silicon for MEMS. Experimental Mechanics 47(1): 37-49. DOI: 10.1007/s11340-006-0405-7.

Crawley EF and De Luis J (1987) Use of piezoelectric actuators as elements of intelligent structures. AIAA Journal 25(10): 1373-1385. DOI: 10.2514/3.9792.

Defaÿ E, Zinck C, Malhaire C, Baboux N and Barbier D (2006) Modified free vibrating beam method for characterization of effective e31 coefficient and leakage resistance of piezoelectric thin films. Review of Scientific Instruments 77(10): 103903. DOI:10.1063/1.2360979.

DeVoe DL (2001) Piezoelectric thin film micromechanical beam resonators. Sensors and Actuators A: Physical 88(3): $263-$ 272. DOI:10.1016/S0924-4247(00)00518-5.

Dietl JM, Wickenheiser AM and Garcia E (2010) A Timoshenko beam model for cantilevered piezoelectric energy harvesters. Smart Materials and Structures 19(5): 055018. DOI: 10.1088/0964-1726/19/5/055018.

Dong W, Lu X, Liu M, Cui Y and Wang J (2007) Measurement on the actuating and sensing capability of a PZT microcantilever. Measurement Science and Technology 18(3): 601-608. DOI: 10.1088/0957-0233/18/3/008.

Dubois MA and Muralt P (1999) Measurement of the effective transverse piezoelectric coefficient $\mathrm{e}_{31, f}$ of AlN and $\mathrm{Pb}\left(\mathrm{Zr}_{x}, \mathrm{Ti}_{1-x}\right) \mathrm{O}_{3}$ thin films. Sensors and Actuators A: Physical 77(2): 106-112. DOI:10.1016/S0924-4247(99)00070-9.

Erturk A and Inman DJ (2008) A Distributed Parameter Electromechanical Model for Cantilevered Piezoelectric Energy Harvesters. Journal of Vibration and Acoustics 130(4): 041002. DOI:10.1115/1.2890402.

Erturk A and Inman DJ (2009) An experimentally validated bimorph cantilever model for piezoelectric energy harvesting from base excitations. Smart Materials and Structures 18(2): 025009. DOI:10.1088/0964-1726/18/2/025009. 
Table 4. Expressions of the parameters appearing in the equations (21)-(23) for the symmetric triple layer bimorph and unimorph piezoelectric beam.

\begin{tabular}{ccc} 
Parameter & triple layer bimorph & unimorph \\
\hline$z_{N}$ & $t_{P}+\frac{t_{S}}{2}$ & $\frac{K_{S} t_{S}^{2}+K_{P} t_{P}\left(2 t_{S}+t_{P}\right)}{2\left(K_{S} t_{S}+K_{P} t_{P}\right)}$ \\
$K I$ & $\frac{W}{12}\left[K_{S} t_{S}^{3}+2 K_{P} t_{P}\left(3 t_{S}^{2}+6 t_{S} t_{P}+4 t_{P}^{2}\right)\right]$ & $\frac{W\left[K_{S}^{2} t_{S}^{4}+K_{P}^{2} t_{P}^{4}+2 K_{S} K_{P} t_{S} t_{P}\left(2 t_{S}^{2}+3 t_{S} t_{P}+2 t_{P}^{2}\right)\right]}{12\left(K_{S} t_{S}+K_{P} t_{P}\right)}$ \\
$K I_{t o t}$ & $K I+\frac{\gamma_{e} \gamma_{m} t_{P}^{3} W}{6 \epsilon_{P}}$ & $K I+\frac{\gamma_{e} \gamma_{m} t_{P}^{3} W}{12 \epsilon_{P}}$ \\
$\Gamma_{m F}$ & $\frac{1}{2} \gamma_{m} W(1-b)\left(t_{P}+t_{S}\right)$ & $\frac{\gamma_{m} W K_{S} t_{S}\left(t_{S}+t_{P}\right)}{2\left(K_{S} t_{S}+K_{P} t_{P}\right)}$ \\
$\Gamma_{e F}$ & $\frac{1}{2} \gamma_{e} W(1-b)\left(t_{P}+t_{S}\right)$ & $\gamma_{e} W K_{S} t_{S}\left(t_{S}+t_{P}\right)$ \\
$K A$ & $W\left(K_{S} t_{S}+2 K_{P}+K_{P} t_{P}\right)$ \\
$\Gamma_{m L}$ & $\gamma_{m} W(1+b)$ & $W\left(K_{S} t_{S}+K_{P} t_{P}\right)$ \\
$\Gamma_{e L}$ & $\gamma_{e} W(1+b)$ & $\gamma_{m} W$ \\
$C_{l}$ & $\frac{2 W \epsilon_{P}}{t_{P}}$ & $\gamma_{e} W$ \\
$\mu_{l}$ & $W\left(2 \rho_{P} t_{P}+\rho_{S} t_{S}\right)$ & $W \epsilon_{P}$ \\
$t_{P}$
\end{tabular}

Gafforelli G, Ardito R and Corigliano A (2015) Improved onedimensional model of piezoelectric laminates for energy harvesters including three dimensional effects. Composite Structures 127: 369-381. DOI:10.1016/j.compstruct.2015.02.065.

Glauvitz N, Blazevic S, Coutu R, Kistler M, Medvedev I and Petkie D (2012) A MEMS Photoacoustic Detector of Terahertz Radiation for Chemical Sensing. Procedia Engineering 47: 730-733. DOI:10.1016/j.proeng.2012.09.251.

Hagedorn P and DasGupta A (2007) Vibrations and Waves in Continuous Mechanical Systems. Chichester, UK: John Wiley \& Sons, Ltd. DOI:10.1002/9780470518434.

Hewa-Kasakarage NN, Kim D, Kuntzman ML and Hall NA (2013) Micromachined Piezoelectric Accelerometers via Epitaxial Silicon Cantilevers and Bulk Silicon Proof Masses. Journal of Microelectromechanical Systems 22(6): 14381446. DOI:10.1109/JMEMS.2013.2262581.

Hindrichsen CC, Almind NS, Brodersen SH, Hansen O and Thomsen EV (2009) Analytical Model of a PZT Thick-Film Triaxial Accelerometer for Optimum Design. IEEE Sensors Journal 9(4): 419-429. DOI:10.1109/JSEN.2009.2014412.

Hindrichsen CC, Almind NS, Brodersen SH, Lou-Møller R, Hansen K and Thomsen EV (2010) Triaxial MEMS accelerometer with screen printed PZT thick film. Journal of Electroceramics 25(2-4): 108-115. DOI:10.1007/s10832010-9597-4.

IEEE 176-1987 (1988) IEEE Standard on Piezoelectricity. Standard on piezoelectricity, IEEE.

Isarakorn D, Briand D, Janphuang P, Sambri A, Gariglio S, Triscone JM, Guy F, Reiner JW, Ahn CH and de Rooij NF (2011) The realization and performance of vibration energy harvesting MEMS devices based on an epitaxial piezoelectric thin film. Smart Materials and Structures 20(2): 025015. DOI:10.1088/0964-1726/20/2/025015.

Ivaldi P, Abergel J, Matheny MH, Villanueva LG, Karabalin RB, Roukes ML, Andreucci P, Hentz S and Defaÿ E (2011) 50 nm thick AlN film-based piezoelectric cantilevers for gravimetric detection. Journal of Micromechanics and Microengineering 21(8): 085023. DOI:10.1088/0960-1317/21/8/085023.

Jackson N, Olszewski OZ, O'Murchu C and Mathewson A (2017) Shock-induced aluminum nitride based MEMS energy harvester to power a leadless pacemaker. Sensors and Actuators A: Physical 264: 212-218. DOI:10.1016/j.sna.2017.08.005.

Jia Y and Seshia AA (2016) Power Optimization by Mass Tuning for MEMS Piezoelectric Cantilever Vibration Energy Harvesting. Journal of Microelectromechanical Systems 25(1): 108-117. DOI:10.1109/JMEMS.2015.2496346.

Karabacak DM, Brongersma SH and Crego-Calama M (2010) Enhanced sensitivity volatile detection with low power integrated micromechanical resonators. Lab on a Chip 10(15): 1976. DOI:10.1039/b926170b.

Karami MA, Bilgen O, Inman DJ and Friswell MI (2011) Experimental and analytical parametric study of single-crystal unimorph beams for vibration energy harvesting. IEEE Transactions on Ultrasonics, Ferroelectrics and Frequency Control 58(7): 1508-1520. DOI:10.1109/TUFFC.2011.1969.

Korayem A, Mashhadian A and Korayem M (2017) Vibration analysis of different AFM cantilever with a piezoelectric layer in the vicinity of rough surfaces. European Journal of Mechanics - A/Solids 65: 313-323. DOI: 10.1016/j.euromechsol.2017.05.003. 
Krommer M (2001) On the correction of the Bernoulli-Euler beam theory for smart piezoelectric beams. Smart Materials and Structures 10(4): 668-680. DOI:10.1088/09641726/10/4/310

Ledermann N, Muralt P, Baborowski J, Forster $\mathrm{M}$ and Pellaux JP (2004) Piezoelectric $\mathrm{Pb}\left(\mathrm{Zr}_{x}, \mathrm{Ti}_{1-x}\right) \mathrm{O}_{3}$ thin film cantilever and bridge acoustic sensors for miniaturized photoacoustic gas detectors. Journal of Micromechanics and Microengineering 14(12): 1650-1658. DOI:10.1088/09601317/14/12/008

Ledermann N, Muralt P, Baborowski J, Gentil S, Mukati K, Cantoni M, Seifert A and Setter N (2003) \{100\}Textured, piezoelectric $\mathrm{Pb}\left(\mathrm{Zr}_{x}, \mathrm{Ti}_{1-x}\right) \mathrm{O}_{3}$ thin films for MEMS: integration, deposition and properties. Sensors and Actuators A: Physical 105(2): 162-170. DOI:10.1016/S09244247(03)00090-6.

Lei A, Xu R, Borregaard LM, Guizzetti M, Hansen O and Thomsen EV (2014) Impedance Based Characterization of a HighCoupled Screen Printed PZT Thick Film Unimorph Energy Harvester. Journal of Microelectromechanical Systems 23(4): 842-854. DOI:10.1109/JMEMS.2013.2295625.

Lim C, He L and Soh A (2001) Three-dimensional electromechanical responses of a parallel piezoelectric bimorph. International Journal of Solids and Structures 38(16): 2833-2849. DOI:10.1016/S0020-7683(00)00186-4.

Littrell R and Grosh K (2012) Modeling and Characterization of Cantilever-Based MEMS Piezoelectric Sensors and Actuators. Journal of Microelectromechanical Systems 21(2): 406-413. DOI:10.1109/JMEMS.2011.2174419.

Lobontiu N and Garcia E (2005) Mechanics of Microelectromechanical Systems. Boston: Kluwer Academic Publishers. DOI:10.1007/b100026.

Luschi L and Pieri F (2015) Design of MEMS mass sensors based of flexural phononic crystals. In: 2015 XVIII AISEM Annual Conference. IEEE, pp. 1-4. DOI: 10.1109/AISEM.2015.7066802.

Luschi L, Pieri F and Iannaccone G (2016) A Simple Method for the Design of 1-D MEMS Flexural Phononic Crystals. IEEE Transactions on Electron Devices 63(10): 4131-4137. DOI: 10.1109/TED.2016.2598757.

Mahameed R, Sinha N, Pisani MB and Piazza G (2008) Dualbeam actuation of piezoelectric AIN RF MEMS switches monolithically integrated with AlN contour-mode resonators. Journal of Micromechanics and Microengineering 18(10): 105011. DOI:10.1088/0960-1317/18/10/105011.

Maurini C, Pouget J and Dell'Isola F (2006) Extension of the Euler Bernoulli model of piezoelectric laminates to include 3D effects via a mixed approach. Computers \& Structures 84(2223): 1438-1458. DOI:10.1016/j.compstruc.2006.01.016.

Olszewski OZ, Houlihan R, Blake A, Mathewson A and Jackson N (2017) Evaluation of Vibrational PiezoMEMS Harvester That Scavenges Energy From a Magnetic Field Surrounding an AC
Current-Carrying Wire. Journal of Microelectromechanical Systems : 1-8DOI:10.1109/JMEMS.2017.2731400.

Piazza G, Abdolvand R, Ho GK and Ayazi F (2004) Voltagetunable piezoelectrically-transduced single-crystal silicon micromechanical resonators. Sensors and Actuators A: Physical 111(1): 71-78. DOI:10.1016/j.sna.2003.10.021.

Qing-Ming Wang and Cross L (1999) Constitutive equations of symmetrical triple layer piezoelectric benders. IEEE Transactions on Ultrasonics, Ferroelectrics and Frequency Control 46(6): 1343-1351. DOI:10.1109/58.808857.

Quenzer H, Drechsler U, Sebastian A, Marauska S, Wagner B and Despont M (2011) Fabrication of conducting AFM cantilevers with AlN-based piezoelectric actuators. Procedia Engineering 25: 665-668. DOI:10.1016/j.proeng.2011.12.164.

Rafique S and Bonello P (2010) Experimental validation of a distributed parameter piezoelectric bimorph cantilever energy harvester. Smart Materials and Structures 19(9): 094008. DOI:10.1088/0964-1726/19/9/094008.

Reissman T, Wickenheiser A and Garcia E (2016) Generalized Solutions of Piezoelectric Vibration-Based Energy Harvesting Structures Using an Electromechanical Transfer Matrix Method. Journal of Vibration and Acoustics 138(4): 041001. DOI:10.1115/1.4033261.

Shibata T, Unno K, Makino E and Shimada S (2004) Fabrication and characterization of diamond AFM probe integrated with PZT thin film sensor and actuator. Sensors and Actuators A: Physical 114(2-3): 398-405. DOI: 10.1016/j.sna.2003.11.025.

Shin S, Kim JP, Sim SJ and Lee J (2008) A multisized piezoelectric microcantilever biosensor array for the quantitative analysis of mass and surface stress. Applied Physics Letters 93(10): 102902. DOI:10.1063/1.2977869.

Smits J and Ballato A (1994) Dynamic admittance matrix of piezoelectric cantilever bimorphs. Journal of Microelectromechanical Systems 3(3): 105-112. DOI: $10.1109 / 84.311560$

Smits J and Choi W (1991) The constituent equations of piezoelectric heterogeneous bimorphs. IEEE Transactions on Ultrasonics, Ferroelectrics and Frequency Control 38(3): 256-270. DOI:10.1109/58.79611.

Smits JG, Dalke SI and Cooney TK (1991) The constituent equations of piezoelectric bimorphs. Sensors and Actuators A: Physical 28(1): 41-61. DOI:10.1016/0924-4247(91)80007C.

Sze K, Yang XM and Fan H (2004) Electric assumptions for piezoelectric laminate analysis. International Journal of Solids and Structures 41(9-10): 2363-2382. DOI: 10.1016/j.ijsolstr.2003.11.018.

Tadmor E and Kosa G (2003) Electromechanical coupling correction for piezoelectric layered beams. Journal of Microelectromechanical Systems 12(6): 899-906. DOI: 10.1109/JMEMS.2003.820286. 
Torri GB, Janssen NMA, Zeng Z, Rottenberg X, Karabacak DM, Vandecasteele M, Hoof CV, Puers R and Tilmans HAC (2014) Piezoelectric transduction of flexural modes in prestressed microbeam resonators. Journal of Micromechanics and Microengineering 24(8): 085014. DOI:10.1088/09601317/24/8/085014.

Weinberg M (1999) Working equations for piezoelectric actuators and sensors. Journal of Microelectromechanical Systems 8(4): 529-533. DOI:10.1109/84.809069.

Wunnicke O (2009) Analytical model of the quasistatic mechanical behavior of galvanic piezoelectric microelectromechanical system switches. Journal of Applied Physics 106(10): 104502. DOI: $10.1063 / 1.3255945$. 